\title{
Combinations of antifungals to effectively kill drug-resistant Candida auris
}

Siddharth Jaggavarapu ${ }^{\mathrm{a}, \mathrm{b}, \mathrm{c}, \mathrm{d}}$, Eileen M. Burd ${ }^{\mathrm{a}, \mathrm{c}}$, and David S. Weiss ${ }^{\mathrm{a}, \mathrm{b}, \mathrm{c}, \mathrm{d}, \#}$

${ }^{a}$ Emory Antibiotic Resistance Center, Atlanta, Georgia, USA

${ }^{\mathrm{b}}$ Emory Vaccine Center, Atlanta, Georgia, USA

${ }^{\mathrm{c}}$ Emory University School of Medicine, Atlanta, Georgia, USA

${ }^{\mathrm{d}}$ Research Service, Atlanta VA Medical Center, Decatur, Georgia, USA

\# Address correspondence to:

David S. Weiss

Emory Antibiotic Resistance Center, 954 Gatewood Road, Atlanta, GA 30329

(404) 727-8214, david.weiss@emory.edu 


\begin{abstract}
Candida auris is an emerging, highly virulent fungal pathogen that is often resistant to multiple classes of antifungals. To overcome this resistance, we investigated the in vitro efficacy of antifungal combinations, finding that amphotericin $\mathrm{B} /$ micafungin resulted in drastically reduced survival $(\sim 5 \operatorname{logs})$ of all 10 isolates tested including 2 that were refractory to each individual drug. These findings suggest that combination therapy with micafungin/amphotericin B may have clinical utility against broadly resistant $C$. auris.
\end{abstract}

\title{
Main Text
}

Resistance to antifungals is increasing and is particularly concerning in the highly pathogenic Candida auris, which is often resistant to multiple classes of antifungal drugs [1]. First discovered in 2009 in the ear canal of a patient in Japan, this fungus has caused outbreaks in multiple healthcare facilities across the world [2-7]. Infections with $C$. auris have high treatment failure rates, resulting in significant mortality [8]. In addition, C. auris can reside and survive on contaminated surfaces for up to one week, making eradication difficult $[9,10]$. Furthermore, routine clinical diagnostics often misidentify $C$. auris as Candida haemulonii, potentially leading to underestimation of its incidence as well as inappropriate treatment [11].

The first line therapy for $C$. auris infections is an echinocandin, with micafungin being the most effective and commonly used $[12,13]$. In cases of echinocandin resistance, the polyene amphotericin B is the recommended second line treatment [13]. However, some isolates of $C$. auris are resistant to all three major classes of antifungals; echinocandins, polyenes, and azoles. While research into developing new antifungal drugs that target $C$. auris is ongoing, the limited 
repertoire of available drugs also necessitates the elucidation of novel methods to more effectively use available antifungals to treat drug-resistant infections [14-16].

We set out to determine the efficacy of combinations of antifungals against 10 isolates of $C$. auris from the FDA/CDC's Antibiotic Resistance (AR) Bank [17]. Due to the lack of clinical breakpoints for $C$. auris, none of the strains have been classified as resistant, susceptible or intermediate to any of the drugs. A recent study of 123 C. auris isolates determined tentative epidemiological cutoff values (ECV), the highest minimum inhibitory concentration (MIC) of the wild-type distribution, for micafungin $(0.25 \mu \mathrm{g} / \mathrm{mL})$, amphotericin $\mathrm{B}(2 \mu \mathrm{g} / \mathrm{mL})$ and voriconazole $(1 \mu \mathrm{g} / \mathrm{mL})$ [1]. Therefore, we used $>0.25 \mu \mathrm{g} / \mathrm{mL}$ as the putative breakpoint for micafungin, $>2 \mu \mathrm{g} / \mathrm{mL}$ for amphotericin $\mathrm{B}$, and $>1 \mu \mathrm{g} / \mathrm{mL}$ for voriconazole, respectively. We first tested the efficacy of voriconazole (Acros, Hampton, NH), micafungin (Biovision, Milpitas, CA) and amphotericin B (Millipore, Burlington, MA) individually in preventing the growth of the 10 C. auris isolates. Serial dilutions of overnight cultures in YPD broth (Sigma, St. Louis, MO) were plated on YPD agar (Sigma, St. Louis, MO) plates to determine the percentage of surviving, resistant cells. The majority of $C$. auris isolates have been reported to be resistant to azoles [18]. Consistent with that finding, voriconazole was ineffective against all 10 isolates even at $4 \mu \mathrm{g} / \mathrm{mL}$, resulting in less than a log reduction in survival (Table 1). Testing of micafungin or amphotericin B revealed that 2 isolates were significantly inhibited by either drug, 3 were susceptible to only micafungin, and 3 were susceptible to only amphotericin B. Two strains were refractory to voriconazole, micafungin, and amphotericin B at the concentrations tested (Table 1). 
The combination of voriconazole and amphotericin B was ineffective at killing any of the strains. In fact, a few isolates that were completely inhibited by amphotericin B, survived better when treated with the combination of voriconazole and amphotericin $\mathrm{B}$, indicative of antagonism (Table 1). Indeed, prior studies have concluded that some azoles and amphotericin B can be antagonistic since these azoles inhibit the biosynthesis of ergosterol, the target of amphotericin B [19].

The combination of voriconazole and micafungin resulted in a $\sim 5 \log$ reduction in survival of 8 of the 10 isolates (Table 1). Importantly, 3 isolates resisted voriconazole and micafungin individually, but were inhibited by their combination, suggesting a synergistic effect of these two antifungals. However, the two isolates that were resistant to the individual effects of voriconazole, micafungin, and amphotericin $\mathrm{B}$, were also uninhibited by voriconazole/micafungin.

Importantly, the combination of micafungin and amphotericin B resulted in a $\sim 5 \log$ reduction in survival of all 10 isolates (Table 1). Notably, this combination was effective against strains 385 and 386 that we classified as resistant to all three individual antifungals tested (Table 1). These results suggest that micafungin/amphotericin B may be able to overcome broadly resistant $C$. auris isolates and offer an effective treatment option using existing drugs and without the need for development of novel antifungals.

Since the current recommended first line treatment for $C$. auris infections is an echinocandin (e.g. micafungin), we tested if initial exposure to micafungin would alter the subsequent efficacy 
of the micafungin/amphotericin B combination, or the current recommended second line treatment of amphotericin B alone. We grew the 5 isolates that were resistant to micafungin overnight in YPD broth containing a sublethal concentration $(0.0625 \mu \mathrm{g} / \mathrm{mL})$ of the drug and subsequently plated onto YPD agar containing micafungin/amphotericin B or amphotericin B alone, to quantify survival. Isolates 383,384 and 387 that were responsive to amphotericin $\mathrm{B}$ monotherapy (Table 1) prior to micafungin treatment were still susceptible to amphotericin B after exposure to sublethal micafungin, suggesting that prior exposure to micafungin did not affect susceptibility to amphotericin B (Table 2). Interestingly, the combination of micafungin/amphotericin B was also still effective against micafungin-pretreated isolates (Table 2). These results suggest that combination therapy with micafungin/amphotericin B may be effective as a second line regimen even after failed treatment with micafungin monotherapy.

The results presented here reveal that the combination of micafungin and amphotericin B has broad efficacy against a collection of $C$. auris clinical isolates, including several strains that we classified as exhibiting resistance to all 3 major antifungal classes. This regimen may have enhanced efficacy as compared to amphotericin B, the current second line therapy for echinocandin resistant strains, and may justify a re-examination of the treatment guidelines. While the results are encouraging, further testing of this combination regimen against clinical isolates will be critical. If these results are representative and broadly applicable, they suggest that the combination of micafungin/amphotericin B may offer an effective option to treat infections caused by highly resistant $C$. auris. 


\section{References}

1. Arendrup, M.C., et al., Comparison of EUCAST and CLSI Reference Microdilution MICs of Eight Antifungal Compounds for Candida auris and Associated Tentative Epidemiological Cutoff Values. Antimicrob Agents Chemother, 2017. 61(6).

2. Calvo, B., et al., First report of Candida auris in America: clinical and microbiological aspects of 18 episodes of candidemia. 2016. 73(4): p. 369-374.

3. Ruiz-Gaitán, A., et al., An outbreak due to Candida auris with prolonged colonisation and candidaemia in a tertiary care European hospital. 2018. 61(7): p. 498-505.

4. Satoh, K., et al., Candida auris sp. nov., a novel ascomycetous yeast isolated from the external ear canal of an inpatient in a Japanese hospital. 2009. 53(1): p. 41-44.

5. Schelenz, S., et al., First hospital outbreak of the globally emerging Candida auris in a European hospital. 2016. 5(1): p. 35.

6. Schwartz, I. and G.J.C.C.D.R. Hammond, Outbreak Detection: First reported case of multidrug-resistant Candida auris in Canada. 2017. 43(7-8): p. 150.

7. Lockhart, S.R., et al., Simultaneous Emergence of Multidrug-Resistant Candida auris on 3 Continents Confirmed by Whole-Genome Sequencing and Epidemiological Analyses. Clin Infect Dis, 2017. 64(2): p. 134-140.

8. Morales-Lopez, S.E., et al., Invasive Infections with Multidrug-Resistant Yeast Candida auris, Colombia. Emerg Infect Dis, 2017. 23(1): p. 162-164.

9. Piedrahita, C.T., et al., Environmental surfaces in healthcare facilities are a potential source for transmission of Candida auris and other Candida species. 2017. 38(9): p. 1107-1109. 
10. Welsh, R.M., et al., Survival, Persistence, and Isolation of the Emerging MultidrugResistant Pathogenic Yeast Candida auris on a Plastic Health Care Surface. J Clin Microbiol, 2017. 55(10): p. 2996-3005.

11. Kathuria, S., et al., Multidrug-Resistant Candida auris Misidentified as Candida haemulonii: Characterization by Matrix-Assisted Laser Desorption Ionization-Time of Flight Mass Spectrometry and DNA Sequencing and Its Antifungal Susceptibility Profile Variability by Vitek 2, CLSI Broth Microdilution, and Etest Method. J Clin Microbiol, 2015. 53(6): p. 1823-30.

12. Lepak, A.J., et al., Pharmacodynamic Optimization for Treatment of Invasive Candida auris Infection. Antimicrob Agents Chemother, 2017. 61(8).

13. Recommendations for treatment of Candida auris infections. 2018; Available from: https://www.cdc.gov/fungal/candida-auris/c-auris-treatment.html.

14. Berkow, E.L., D. Angulo, and S.R. Lockhart, In Vitro Activity of a Novel Glucan Synthase Inhibitor, SCY-078, against Clinical Isolates of Candida auris. Antimicrob Agents Chemother, 2017. 61(7).

15. Singh S, U.P., Alqarihi A, Elhassan H, French S, Lockhart SR, et al, The NDV-3A vaccine protects mice from multidrug resistant Candida auris infection. bioRxiv 2018. 465096.

16. Tetz, G., et al., In Vitro Activity of a Novel Antifungal Compound, MYC-053, against Clinically Significant Antifungal-Resistant Strains of Candida glabrata, Candida auris, Cryptococcus neoformans, and Pneumocystis spp. Antimicrob Agents Chemother, 2019. 63(4). 
17. CDC \& FDA Antibiotic Resistance Isolate Bank. 2019; Available from:

https://wwwn.cdc.gov/ARIsolateBank/Panel/PanelDetail?ID=2.

18. Chowdhary, A., et al., A multicentre study of antifungal susceptibility patterns among 350 Candida auris isolates (2009-17) in India: role of the ERG11 and FKS1 genes in azole and echinocandin resistance. J Antimicrob Chemother, 2018. 73(4): p. 891-899.

19. Sugar, A.M., Use of amphotericin B with azole antifungal drugs: what are we doing? Antimicrob Agents Chemother, 1995. 39(9): p. 1907-12. 
Table 1. Survival of $C$. auris isolates plated on antifungal drugs and combinations ${ }^{a}$

\begin{tabular}{|c|c|c|c|c|c|c|c|}
\hline Isolate & no drug & Vori & Mica & Amp B & $\mathbf{V + A}$ & $\mathbf{V + M}$ & $\mathbf{M}+\mathbf{A}$ \\
\hline $\mathbf{3 8 1}$ & 300,000 & 400,000 & 0 & 0 & 1,000 & 0 & 0 \\
\hline $\mathbf{3 8 2}$ & 700,000 & 700,000 & $<10^{b}$ & 0 & 500,000 & 0 & 0 \\
\hline $\mathbf{3 8 3}$ & $1,200,000$ & 200,000 & 400,000 & $<10^{b}$ & 400,000 & $<10^{b}$ & 0 \\
\hline $\mathbf{3 8 4}$ & 600,000 & 600,000 & 600,000 & $<10^{b}$ & 500,000 & $<10^{b}$ & $<10^{b}$ \\
\hline $\mathbf{3 8 5}$ & 300,000 & 200,000 & 200,000 & 200,000 & 600,000 & 10,000 & $<10^{b}$ \\
\hline $\mathbf{3 8 6}$ & 800,000 & $1,200,000$ & 300,000 & 500,000 & 700,000 & 100,000 & $<10^{b}$ \\
\hline $\mathbf{3 8 7}$ & 700,000 & 700,000 & 100,000 & $<10^{b}$ & 200,000 & 0 & $<10^{b}$ \\
\hline $\mathbf{3 8 8}$ & 500,000 & 300,000 & 2 & 70,000 & 250,000 & 4 & $<10^{b}$ \\
\hline $\mathbf{3 8 9}$ & 800,000 & 500,000 & $<10^{b}$ & 400,000 & 300,000 & 2 & 2 \\
\hline $\mathbf{3 9 0}$ & 500,000 & 600,000 & 0 & 500,000 & 500,000 & 10 & $<10^{b}$ \\
\hline
\end{tabular}

${ }^{a}$ Surviving CFUs are shown following plating of the indicated $C$. auris isolates on no drug, voriconazole (Vori, V; $4 \mu \mathrm{g} / \mathrm{mL}$ ), micafungin (Mica, M; $0.25 \mu \mathrm{g} / \mathrm{mL}$ ), amphotericin B (Amp $\mathrm{B}, \mathrm{A} ; 2 \mu \mathrm{g} / \mathrm{mL}$ ), or their combinations. Cells within the Table are colored in a gradient from highest CFUs (red) to lowest (green).

${ }^{b}$ CFUs could not be determined exactly since dense growth was observed at the highest concentration of bacteria, but no growth was observed at the next dilution. 
Table 2. Survival of $C$. auris after initial exposure to sublethal micafungin ${ }^{a}$

\begin{tabular}{|c|c|c|c|c|}
\hline Isolate & no AB & Mica & AmpB & M+A \\
\hline $\mathbf{3 8 3}$ & 100,000 & 200,000 & 10 & 0 \\
\hline $\mathbf{3 8 4}$ & 60,000 & 60,000 & 0 & 0 \\
\hline $\mathbf{3 8 5}$ & 40,000 & 20,000 & 2,100 & 0 \\
\hline $\mathbf{3 8 6}$ & 80,000 & 20,000 & 20,000 & 0 \\
\hline $\mathbf{3 8 7}$ & 40,000 & 10,000 & 0 & 0 \\
\hline
\end{tabular}

${ }^{a}$ All isolates were initially grown overnight in the presence of $0.0625 \mu \mathrm{g} / \mathrm{mL}$ micafungin. Subsequently, isolates were plated onto no drug ("no AB") or the indicated drugs at concentrations of $0.25 \mu \mathrm{g} / \mathrm{mL}$ micafungin (Mica, M) and $/$ or $2 \mu \mathrm{g} / \mathrm{mL}$ amphotericin B (AmpB, A). Cells within the Table are colored in a gradient from the highest CFUs (red) to the lowest (green). 